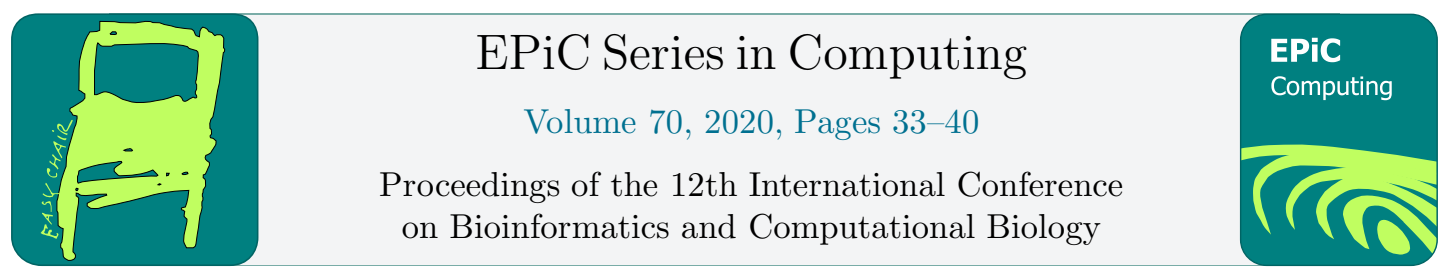

\title{
Bifurcation Analysis of a Mathematical Model of Tumor Growth in MCF-7 Breast Cancer Cell Line
}

\author{
Hsiu-Chuan Wei ${ }^{1}$ \\ Feng Chia University, Taichung, Taiwan \\ hsiucwei@fcu.edu.tw
}

\begin{abstract}
Breast cancer is the second leading cause of cancer death for women worldwide. In this study, a previously published mathematical model of breast cancer in MCF-7 cell line is considered. The interaction among tumor cells, estradiol, natural killer (NK) cells, cytotoxic T lymphocytes (CTLs) or CD8+ T cells, and white blood cells (WBCs), is described by ordinary differential equations (ODEs). The system exhibits three coexisting stable equilibrium points which resemble the 3 E's (elimination, equilibrium, and escape) of cancer immunoediting. In this paper, a numerical method based on adaptive grid method is employed for bifurcation analysis of the mathematical model. Bifurcation analysis is performed for some important parameters for which changes in value result in changes in the stability of steady states. The results obtained from the bifurcation analysis may provide useful information about treatment strategy in further studies.
\end{abstract}

\section{Introduction}

Breast cancer is the second most common cancer in women worldwide [7,8], and it is a heterogeneous disease. Approximately $75 \%$ of breast cancer cases are ER+ [14]. The MCF-7 cell line is a common in vitro model used for studies on ER+ breast cancer. Simms et al. [16] have presented a mathematical model of cell cycle progression and applied the mathematical model to the MCF-7 cell line to investigate cell proliferation and the effect of treatment using tamoxifen. Oke et al. [13] have proposed a mathematical model for ER+ breast cancer with optimal control analysis. Wei [20] has introduced a mathematical model for tumor growth in MCF-7 breast cancer cell line with interaction among the cancer cells and immune cells. This work is motivated by the mathematical model developed by Wei [20]. The model was based on several experiments conducted by Nawata et al. [12], Caragine et al. [3], Müller et al. [10], Chen et al. [4], using MCF-7 breast cancer cells. The mathematical model exhibits multistability where the tumor free, microscopic tumor, and large tumor steady states are stable. The phenomena resemble the 3 E's of cancer immunoediting [6, 11]. Numerical simulation using direct integration has shown that immune system strength is important in determining whether or not an immune system is able to eliminate a small tumor or produce long-term dormancy.

Bifurcation analysis is a powerful tool to study the changes in dynamics as parameter values vary. Identifying the parameters for which changes in value lead to changes in dynamics of the 
mathematical model can provide useful information about the treatment strategy. Recently, numerical methods for bifurcation analysis have been developed for ODE systems with periodically pulsed therapies $[18,19,21]$. These numerical methods are based on adaptive grid technique for bifurcations of fixed points in ODE systems with periodically pulsed inputs. In this paper, the numerical methods is to be applied to the mathematical model for the identification of periodic solutions and bifurcation curves where a fixed point changes its stability. Computation of bifurcation curves provides an efficient means to find the sets of parameter values at which the system changes its asymptotic behavior. This can help identify important parameters that affect tumor population dynamics and manage treatment strategy.

To provide details, the numerical approach for locating periodic solutions is presented in Section 2. Numerical examples and discussion are given in Section 3. Finally, a brief conclusion is made in Section 4.

\section{Numerical methods for locating the periodic solutions}

\subsection{Mathematical model}

The mathematical model proposed by Wei [20] is as follows:

$$
\begin{aligned}
\frac{d T}{d t}= & T\left(a+\frac{c E T}{1+\alpha_{1} E+\beta_{1} T^{2}}\right)(1-T / K)-\frac{p_{1} T N^{2}}{1+\alpha_{2} T+\beta_{2} N^{2}} \\
& -\frac{p_{6} T^{2} L}{1+\alpha_{6} T^{2}+\beta_{6} L}, \\
\frac{d N}{d t}= & e C-f N-p_{2} N T+\frac{p_{3} N T}{1+\alpha_{3} T+\beta_{3} N}, \\
\frac{d L}{d t}= & \left(p_{4} L_{N}+\frac{p_{5} I}{\alpha_{4}+I} L\right)\left(1-L / K_{L}\right) \frac{T}{\alpha_{5}+T}-d L, \\
\frac{d C}{d t}= & \alpha-\beta C, \\
E(t)= & \tilde{E}(t-n \tau), \quad t \in[n \tau,(n+1) \tau),
\end{aligned}
$$

where $E(t)$ is a periodic function and $t$ is in days. The model consists of five state variables: the MCF-7 tumor cell population $T$ (cells), the circulating level of estradiol $E$ (pmol/L), NK cell population $N$ (cells/L), CTL (or CD8+ T) cell population $L$ (cells/L), and WBC population $C$ (cells/L). Parameters $a$ and $K$ are growth rate and carrying capacity of tumor cells, respectively; $I$ is the concentration of interleukin 2(IL-2) and $p_{5}$ is the maximum growth rate of CTLs induced by IL-2; $L_{N}, p_{4}, K_{L}$, and $d$ represent naive CTL population, the fraction of naive CTLs that become activated, the carrying capacity of CTLs, and the death rate of CTLs, respectively; $e$, $f$, and $p_{2}$ are fraction of WBCs that become NK cells, death rate of NK cells, and NK cell inactivation rate by tumor cells, respectively; $p_{3}$ is related to NK cell recruitment rate and $c$ is related to the growth rate of tumor cells induced by circulating level of estradiol; $\alpha_{i}$ for $i=1 \cdots 6$ and $\beta_{i}$ for $i=1,2,3,6$ are related to half saturation constants; $p_{1}$ and $p_{6}$ are rates of NK- and CTL-induced tumor death, respectively; $\alpha$ and $\beta$ are production rate and death rate of WBCs, respectively. The parameter values are summarized and shown in Table 1. 
Table 1: Parameter values in Eqs. (1)-(5).

\begin{tabular}{|c|c|c|}
\hline Parameter & Value & Units \\
\hline$a$ & 0.3 & Day $^{-1}$ \\
\hline$c$ & $1.93 \times 10^{-6}$ & $\mathrm{~L} \mathrm{Cell}^{-1} \mathrm{Day}^{-1} \mathrm{pmol}^{-1}$ \\
\hline$\alpha_{1}$ & 0.507 & $\mathrm{~L} \mathrm{pmol}^{-1}$ \\
\hline$\beta_{1}$ & $7.08 \times 10^{-8}$ & Cell $^{-1}$ \\
\hline$K$ & $10^{9}$ & Cell \\
\hline$p_{1}$ & $8.7 \times 10^{-4}$ & $\mathrm{~L}^{2} \mathrm{Cell}^{-2} \mathrm{Day}^{-1}$ \\
\hline$\alpha_{2}$ & $7 \times 10^{6}$ & Cell $^{-1}$ \\
\hline$\beta_{2}$ & $5.4 \times 10^{-5}$ & $\mathrm{~L}^{2} \mathrm{Cell}^{-2}$ \\
\hline$\beta$ & $6.3 \times 10^{-3}$ & Day $^{-1}$ \\
\hline$\alpha$ & $3.6 \times 10^{7}$ & Cell L ${ }^{-1}$ Day $^{-1}$ \\
\hline$e$ & 0.00486 & Day $^{-1}$ \\
\hline$f$ & 0.0693 & Day $^{-1}$ \\
\hline$p_{2}$ & $3.42 \times 10^{-6}$ & Cell Day ${ }^{-1}$ \\
\hline$p_{3}$ & $1.87 \times 10^{-8}$ & Cell $^{-1}$ Day $^{-1}$ \\
\hline$\alpha_{3}$ & $1.6 \times 10^{-5}$ & Cell $^{-1}$ \\
\hline$\beta_{3}$ & 3.27 & $\mathrm{~L} \mathrm{Cell}^{-1}$ \\
\hline$p_{6}$ & $2.04 \times 10^{-3}$ & $\mathrm{~L} \mathrm{Cell}^{-2}$ Day $^{-1}$ \\
\hline$\alpha_{6}$ & 0.268 & Cell $^{-2}$ \\
\hline$\beta_{6}$ & 4343 & $\mathrm{~L}$ Cell $^{-1}$ \\
\hline$L_{N}$ & $2.3 \times 10^{8}$ & Cell L $\mathrm{L}^{-1}$ \\
\hline$K_{L}$ & $8 \times 10^{8}$ & Cell L $\mathrm{L}^{-1}$ \\
\hline$p_{4}$ & $9 \times 10^{-5}$ & Day $^{-1}$ \\
\hline$I$ & $2.3 \times 10^{-11}$ & $\mathrm{~g} \mathrm{~L}^{-1}$ \\
\hline$\alpha_{4}$ & $2.3 \times 10^{-11}$ & $\mathrm{~g} \mathrm{~L}^{-1}$ \\
\hline$p_{5}$ & 4.14 & $\mathrm{~L} \mathrm{Cell}^{-2} \mathrm{Day}^{-1}$ \\
\hline$d$ & 0.41 & Day $^{-1}$ \\
\hline$\alpha_{5}$ & 1000 & Cell \\
\hline
\end{tabular}

\subsection{Stability of the tumor-free equilibrium}

The tumor-free equilibrium $E_{0}$ of Eqs. (1)-(5) is $(T, N, L, C)=\left(0, \frac{e \alpha}{f \beta}, 0, \alpha / \beta\right)$. Let $C_{p}=\alpha / \beta$ and thus $N_{p}=e C_{p} / f$. Then, the Jacobian matrix at $E_{0}$ is

$$
J\left(E_{0}\right)=\left[\begin{array}{cccc}
a-\frac{p_{1} N_{p}^{2}}{1+\beta_{2} N_{p}^{2}} & 0 & 0 & 0 \\
-p_{2} N_{p}+\frac{p_{3} N_{p}}{1+\beta_{3} N_{p}} & -f & 0 & e \\
p_{4} / \alpha_{5} & 0 & -d & 0 \\
0 & 0 & 0 & -\beta
\end{array}\right] .
$$

The tumor-free equilibrium is stable if $a<\frac{p_{1} N_{p}^{2}}{1+\beta_{2} N_{p}^{2}}$.

\subsection{Numerical methods for locating periodic solutions}

Since the circulation estradiol level $E(t)$ is a periodic function with period $\tau=29$, the system, Eqs. (1)-(5), has equilibrium point only when $T=0$. However, the system may have periodic solutions of period $\tau$. In fact, numerical simulation conducted by Wei [20] has shown that the system has stable periodic solutions with small oscillations in population levels. To find periodic solutions, it may be assumed that $C(t)$ has reached its equilibrium population level and thus the system, Eqs. (1)-(5), is reduced to Eqs. (1)-(3) and (5) with $C=\alpha / \beta$. Let 
$F(t)=(T(t), N(t), L(t))$. A periodic solution satisfies $F(\tau)-F(0)=0$ which is to be solved by Newton's method.

Let $T(0)=T_{0}, N(0)=N_{0}$, and $L(0)=L_{0}$, and let the Jacobian matrix of the right hand side of Eqs. (1)-(3) be $J$. The Jacobian matrix

$$
A(t)=\left[\begin{array}{ccc}
\partial T / \partial T_{0} & \partial T / \partial N_{0} & \partial T / \partial L_{0} \\
\partial N / \partial T_{0} & \partial N / \partial N_{0} & \partial N / \partial L_{0} \\
\partial L / \partial T_{0} & \partial L / \partial N_{0} & \partial L / \partial L_{0}
\end{array}\right] .
$$

satisfies

$$
A^{\prime}(t)=J A(t), \quad A(0)=I_{3 \times 3} .
$$

Recall that the system has a stable tumor-free equilibrium and two stable periodic solutions with small oscillations for the set of parameter values shown in Table 1. This also implies that the system has two unstable periodic solutions. Locating each of the periodic solutions requires solving (8) and an initial guess for Newton's method. In this paper, the initial guess is obtained by finding all the equilibrium points for Eqs. (1)-(3) with $E(t)$ replaced by its average value over $[0, \tau]$, which is $\bar{E}=\int_{0}^{\tau} E(t) d t / \tau$. Note that these initial guesses satisfy

$$
\begin{aligned}
0= & \left(a+\frac{c \bar{E} T}{1+\alpha_{1} \bar{E}+\beta_{1} T^{2}}\right)(1-T / K)-\frac{p_{1} N^{2}}{1+\alpha_{2} T+\beta_{2} N^{2}} \\
& -\frac{p_{6} T L}{1+\alpha_{6} T^{2}+\beta_{6} L}, \\
0= & e C-f N-p_{2} N T+\frac{p_{3} N T}{1+\alpha_{3} T+\beta_{3} N}, \\
0= & \left(p_{4} L_{N}+\frac{p_{5} I}{\alpha_{4}+I} L\right)\left(1-L / K_{L}\right) \frac{T}{\alpha_{5}+T}-d L .
\end{aligned}
$$

The variable $L$ can be expressed in terms of $T$ from Eq. (11) and then $N$ can be written in terms of $T$ and $L$ from Eq. (9). Next, consider an evenly spaced grid over $[0, \log K]$. Let $x_{i}$ be the $i$ th grid point in $[0, \log K]$ and $T_{i}=10^{x_{i}}$. Then, evaluate the right hand side of Eq. (10), and an equilibrium point might exist for $T \in\left(T_{i}, T_{i+1}\right)$ if the right hand sides of Eq. (10) have different signs at $T_{i}$ and $T_{i+1}$. Use a $T$ value in $\left(T_{i}, T_{i+1}\right)$, an $L$ value obtained from (11), and an $N$ value obtained from Eq. (9) as an initial guess for Newton's method to solve Eqs. (9)-(11). The resulting equilibrium points are then used as initial guesses for solving $F(\tau)-F(0)=0$.

\section{Numerical examples and discussion}

It has been reported that a level of ER greater than 5 fmoles/mg protein indicates an ER positive breast cancer $[2,15]$. The breast cancer patients may have a level of ER greater than $300 \mathrm{fmoles} / \mathrm{mg}$ [1] depending on individual variation. This implies that tumor cell proliferation induced by estradiol stimulation varies in individual patients and $c$ values may vary largely in $\mathrm{ER}+$ breast cancer patients. Recent immunotherapy based on enhancing $\mathrm{T}$ cell response to tumor cells, such as anti PD-1 antibody therapy, has shown promising response rates [5]. The parameter $p_{6}$ is related to T cell response to tumor cells. Thus, parameters $c$ and $p_{6}$ are used as bifurcation parameters in the first numerical example.

Fig. 1(a) shows the regions in which the system has different asymptotic properties on the parameter domain $\left[10^{-6}, 10^{-3}\right] \times\left[10^{-3}, 4 \times 10^{-2}\right]$. Let $E_{1}$ denote the small tumor periodic solution and $E_{2}$ denote the large tumor periodic solution. Note that $E_{0}$, and $E_{1}$, and $E_{2}$ are 
stable solutions. The immune system is able to eliminate or regress a large tumor when CTLs display strong responses $\left(p_{6}>0.33\right)$ against tumors. Coexistence of $E_{0}, E_{1}$, and $E_{3}$ in a large region shows that a normal immune system is able to eliminate a small tumor or control a small tumor producing long-term dormancy. There are two regions in which $E_{0}$ and $E_{2}$ exist. Fig. 1 (b) shows that the immune system is able to eliminate a tumor of moderate size $\left(2 \times 10^{8}\right.$ cells $)$ in the region with small ER levels while Fig. 1(c) shows that a small tumor of $2 \times 10^{6}$ cells grows to its carrying capacity in the region with small CTL response.

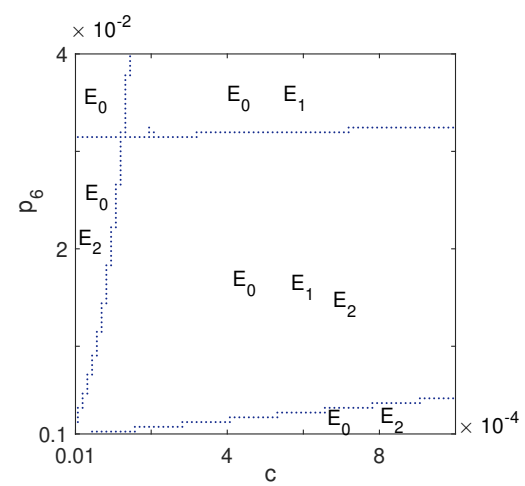

(a)

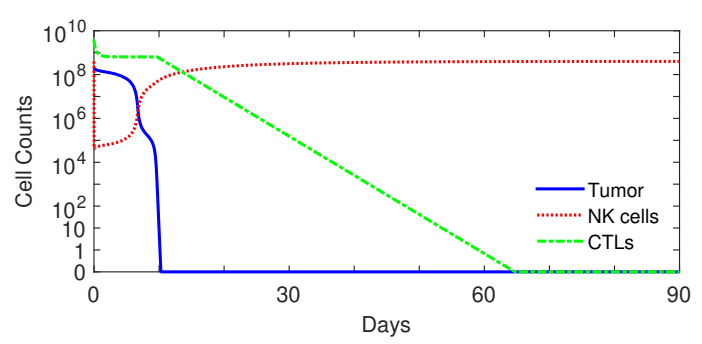

(b)

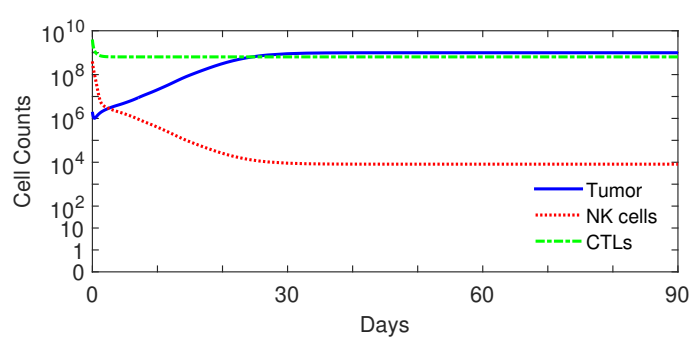

(c)

Figure 1: (a) Bifurcation diagram using $c$ and $p_{6}$ as bifurcation parameters. Cell population dynamics for (b) $c=4 \times 10^{-4}$ and $p_{6}=10^{-3}$ with initial condition $(T(0), N(0), L(0))=$ $\left(2 \times 10^{6}, 4 \times 10^{8}, 4 \times 10^{9}\right)$, and $(\mathrm{c}) c=2 \times 10^{-6}$ and $p_{6}=10^{-3}$ with initial condition $(T(0), N(0), L(0))=\left(2 \times 10^{8}, 4 \times 10^{8}, 4 \times 10^{9}\right)$.

Combination therapy with two or more drugs for cancer treatment has been shown to be more effective than monotherapy [22]. It can achieve additive or synergistic effects with lower doses or lower toxicity drugs. Recent clinical trials have suggested combination therapy with a BCL-2 inhibitor and tamoxifen in ER positive breast cancer [9]. Tamoxifen is usually considered to be an estrogen antagonist to block estrogen receptors in mammary gland. BCL-2 is known to inhibit cell apoptosis. A BCL-2 inhibitor inhibits the activity of BCL-2 and restores apoptosis of tumor cells. Assuming that a BCL-2 inhibitor can enhance apoptosis of tumor cells so that the tumor grows less progressively upon the administration of a BCL-2 inhibitor. Consequently, this results in a smaller $a$ values. In this example, parameter $c$ and $a$ are used as bifurcation parameters. 


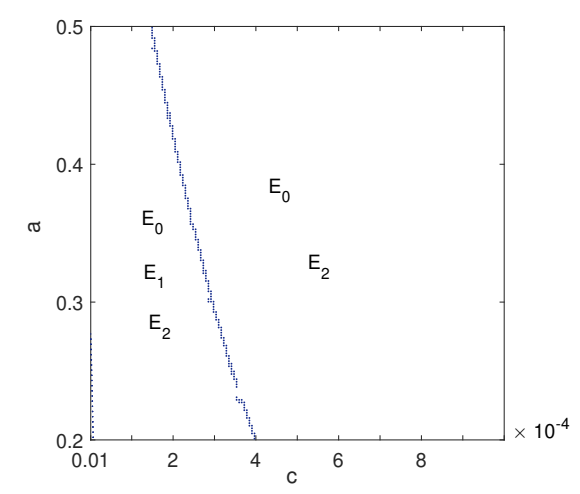

Figure 2: Bifurcation diagram using $c$ and $a$ as bifurcation parameters.

Fig. 2 shows the bifurcation diagram on the parameter domain $\left[10^{-6}, 10^{-3}\right] \times[0.2,0.5]$. The system has stable solutions $E_{0}$ and $E_{2}$ in the region where the tumor has a high ER level. A small tumor will grow to its carrying capacity. The situation is similar to that shown in Fig. 1(c). The use of drugs that block estrogen receptors can lower $c$ values and consequently decrease tumor proliferation induced by estrogen stimulation. As the $c$ value decreases, it brings the system to the region where $E_{0}, E_{1}$, and $E_{2}$ exist. The immune system is able to eliminate a small tumor or produce long term dormancy. Interestingly note that reducing the $a$ value can change the system's asymptotic behavior only when $c \in\left[2.5 \times 10^{-4}, 4 \times 10^{-4}\right]$. This implies that drugs, such as a BCL-2 inhibitor, that can reduce tumor growth rate may not be effective when used alone. However, reducing tumor growth rate combines with reducing ER levels may produce synergistic effect. In fact, Vaillant et al. [17] have reported that the administration of ABT-199, a BCL-2 inhibitor, alone is ineffective in ER+ breast cancer but combining ABT-199 with tamoxifen can produce synergistic effect.

\section{Conclusion}

This paper which is based on a mathematical model previously developed by Wei [20] studies bifurcations of the model. The condition for which the tumor-free equilibrium is stable has been proven. Then, numerical techniques based on an adaptive grid method have been proposed to perform bifurcation analysis. Numerical simulation has shown that enhancing CTL response to tumor cells can help the immune system to control a large tumor. Cancer treatment such as tumor infiltrating lymphocyte (TIL) therapy together with checkpoint inhibitor (such as antiPD-1 or anti-PD-L1 drugs) may be used to enhance the anti-tumor activity of CTLs. Numerical simulation has also shown that decreases in tumor cell growth rate may be ineffective when the tumor has high ER levels. Combination therapy, such as BCL-2 inhibitor with tamoxifen that slows tumor growth and reduces ER levels may produce synergistic effects.

\section{Acknowledgments}

This research work was supported by the Ministry of Science and Technology of Taiwan under the grant MOST108-2115-M-035-006. 


\section{References}

[1] W. R. Bezwoda, J. D. Esser, R. Dansey, I. Kessel, and M. Lange. The value of estrogen and progesterone receptor determinations in advanced breast cancer. estrogen receptor level but not progesterone receptor level correlates with response to tamoxifen. Cancer 68 (1991), 867-872.

[2] S. C. Brooks, D. E. Saunders, A. Singhakowinta, and V. K. Vaitkevicius. Relation of tumor content of estrogen and progesteron receptors with response of patient to endocrine therapy. Cancer 46 (1980), 2775-2778.

[3] T. A. Caragine, M. Imai, A. B. Frey, and S. Tomlinson. Expression of rat complement control protein Crry on tumor cells inhibits rat natural killer cell-mediated cytotoxicity. Blood 100 (2002), 6895-6902.

[4] J. Chen, E. Hui, T. Ip, and L. U. Thompson. Dietary flaxseed enhances the inhibitory effect of tamoxifen on the growth of estrogen-dependent human breast cancer (mcf-7) in nude mice. Clin Cancer Res 10 (2004), 7703-7711.

[5] L. Chen and X. Han. Anti-pd-1/pd-11 therapy of human cancer: Past, present, and future. J Clin Invest 125 (2015), 3384-3391.

[6] G. P. Dunn, A. T. Bruce, H. Ikeda, L. J. Old, and R. D. Schreiber. Cancer immunoediting: from immunosurveillance to tumor escape. Nat Immunol 3 (2002), 991.

[7] D. R. Jutagir, B. B. Blomberg, C. S. Carver, S. C. Lechner, K. R. Timpano, L. C. Bouchard, , L. M. Gudenkauf, J. M. Jacobs, A. Diaz, S. K. Lutgendorf, S. W. Cole, A. S. Heller, and M. H. Antoni. Social well-being is associated with less pro-inflammatory and pro-metastatic leukocyte gene expression in women after surgery for breast cancer. Breast Cancer Res Treat 165 (2017), 169-180.

[8] S. Katkuri and M. Gorantla. Awareness about breast cancer among women aged 15 years and above in urban slums: a cross sectional study. Int J Community Med Public Health 5 (2018), 929-932.

[9] S. W. Lok, J. R. Whittle, F. Vaillant, C. E. Teh, L. L. Lo, A. N. Policheni, A. R. T. Bergin, J. Desai, S. Ftouni, L. C. Gandolfo, D. Liew, H. K. Liu, G. B. Mann, K. Moodie, A. Murugasu, B. Pal, A. W. Roberts, M. A. Rosenthal, K. Shackleton, M. J. Silva, Z. R. Siow, G. K. Smyth, L. Taylor, A. Travers, B. Yeo, M. M. Yeung, A. Z. Bujak, S. J. Dawson, D. H.D. Gray, J. E. Visvader, and G. J. Lindeman. A phase $1 \mathrm{~b}$ dose-escalation and expansion study of the bcl2 inhibitor venetoclax combined with tamoxifen in er and bcl2 positive metastatic breast cancer. Cancer Discov 9 (2019), 354-369.

[10] M. R. Müller, F. Grünebach, A. Nencioni, and P. Brossart. Transfection of dendritic cells with RNA induces cd4-and cd8-mediated $\mathrm{T}$ cell immunity against breast carcinomas and reveals the immunodominance of presented T cell epitopes. J Immunol 170 (2003), 5892-5896.

[11] D. Mittal, M. M. Gubin, R. D. Schreiber, and M. J. Smyth. New insights into cancer immunoediting and its three component phases - elimination, equilibrium and escape. Curr Opin Immunol 27 (2014), 16-25.

[12] H. Nawata, M. T. Chong, D. Bronzert, and M. E. Lippman. Estradiol-independent growth of a subline of MCF-7 human breast cancer cells in culture. J Biol Chem 256 (1981), 6895-6902.

[13] S. I. Oke, M. B. Matadi, and S. So. Xulu. Optimal control analysis of a mathematical model for breast cancer. Math Comput Appl 23 (2018), https://doi.org/10.3390/mca23020021.

[14] A. Pawlik, M. Slomińska-Wojewódzka, A, and Herman-Antosiewicz. Sensitization of estrogen receptor-positive breast cancer cell lines to 4-hydroxytamoxifen by isothiocyanates present in cruciferous plants. Eur J Nutr 55 (2016), 1165-1180.

[15] B. Ramsey, T. Bai, A. H. Newell, M. Troxell, B. Park, S. Olson, E. Keenan, and S. W. Luoh. Grb7 protein over-expression and clinical outcome in breast cancer. Breast Cancer Res Treat 127 (2011), 659-669.

[16] K. Simms, N. Beanand, and A. Koerber. A mathematical model of cell cycle progression applied 
to the mcf-7 breast cancer cell line. Bull Math Biol 74 (2012), 736-767.

[17] F. Vaillant, D. Merino, L. Lee, K. Breslin, B. Pal, M. E. Ritchie, G. K. Smyth, M. Christie, L. J. Phillipson, C. J. Burns, G. B. Mann, J. E. Visvader, and G. J.Lindeman. Targeting bcl-2 with the bh3 mimetic abt-199 in estrogen receptor-positive breast cancer. Cancer Cell 24 (2013), 120-129.

[18] H. C. Wei. A modified numerical method for bifurcations of fixed points of ode systems with periodically pulsed inputs. Appl Math Comput 236 (2014), 373-383.

[19] H. C. Wei. A numerical study of a mathematical model of pulsed immunotherapy for super-ficial bladder cancer. Jpn J Ind Appl Math 30 (2013), 441-452.

[20] H. C. Wei. Mathematical modeling of tumor growth: the mcf-7 breast cancer cell line. Math Biosci Eng 16 (2019), 6512-6535.

[21] H. C. Wei and J. T. Lin. Periodically pulsed immunotherapy in a mathematical model of tumorimmune interaction. Internat J Bifur Chaos Appl Sci Engrg 23 (2013), 135006813 pp.

[22] X. Zhang, L. Li, C. Li, H. Zheng, H. Song, F. Xiong, T. Qiu, and J. Yang. Cisplatin-crosslinked glutathione-sensitive micelles loaded with doxorubicin for combination and targeted therapy of tumors. Carbohydr Polym 155 (2017), 407-415. 